\title{
Structural and functional analysis of the
} finished genome of the recently isolated toxic Anabaena sp. WA102

\author{
Nathan M. Brown' ${ }^{1}$, Ryan S. Mueller', Jonathan W. Shepardson' , Zachary C. Landry', Jeffrey T. Morré2, \\ Claudia S. Maier ${ }^{2}$, F. Joan Hardy ${ }^{3}$ and Theo W. Dreher ${ }^{1,4^{*}}$
}

\begin{abstract}
Background: Very few closed genomes of the cyanobacteria that commonly produce toxic blooms in lakes and reservoirs are available, limiting our understanding of the properties of these organisms. A new anatoxin-a-producing member of the Nostocaceae, Anabaena sp. WA102, was isolated from a freshwater lake in Washington State, USA, in 2013 and maintained in non-axenic culture.

Results: The Anabaena sp. WA102 5.7 Mbp genome assembly has been closed with long-read, single-molecule sequencing and separately a draft genome assembly has been produced with short-read sequencing technology. The closed and draft genome assemblies are compared, showing a correlation between long repeats in the genome and the many gaps in the short-read assembly. Anabaena sp. WA102 encodes anatoxin-a biosynthetic genes, as does its close relative Anabaena sp. AL93 (also introduced in this study). These strains are distinguished by differences in the genes for light-harvesting phycobilins, with Anabaena sp. AL93 possessing a phycoerythrocyanin operon. Biologically relevant structural variants in the Anabaena sp. WA102 genome were detected only by long-read sequencing: a tandem triplication of the $a n a B C D$ promoter region in the anatoxin-a synthase gene cluster (not triplicated in Anabaena sp. AL93) and a 5-kbp deletion variant present in two-thirds of the population. The genome has a large number of mobile elements (160). Strikingly, there was no synteny with the genome of its nearest fully assembled relative, Anabaena sp. 90.
\end{abstract}

Conclusion: Structural and functional genome analyses indicate that Anabaena sp. WA102 has a flexible genome. Genome closure, which can be readily achieved with long-read sequencing, reveals large scale (e.g., gene order) and local structural features that should be considered in understanding genome evolution and function.

Keywords: Anabaena, Anatoxin-a, Synteny, Cyanobacteria, Mobilome, Long-read sequencing, PacBio, Illumina, Tandem repeat, Structural variation

\section{Background}

Anabaena (some isolates are also named Dolichospermum [1]) are filamentous, nitrogen-fixing cyanobacteria that often form blooms in eutrophic water bodies. Traditionally, they have been studied as models of multicellular development in bacteria [2]. Their ability to fix both

\footnotetext{
*Correspondence: theo.dreher@oregonstate.edu

1 Department of Microbiology, Oregon State University, 226 Nash Hall, 97331 Corvallis, OR, USA

${ }^{4}$ Center for Genome Research and Biocomputing, Oregon State University, Corvallis, OR, USA

Full list of author information is available at the end of the article
}

carbon and nitrogen makes them a key part of the biogeochemical cycle. Further, they can produce a range of bioactive secondary metabolites, which have been shown to threaten public health whenever toxic blooms occur in drinking or recreational water bodies $[3,4]$.

Anatoxin-a is one of the most toxic secondary metabolites produced by Anabaena species [5]. It acts as a nicotinic acetylcholine receptor agonist in animals, paralyzing muscles and causing death by asphyxiation [6]. The toxin is synthesized via a polyketide synthase (PKS) pathway encoded by a cluster of at least eight genes [7]. Anatoxin-a 
is known to be synthesized by five genera of Cyanobacteria: Anabaena (Dolichospermum), Oscillatoria, Aphanizomenon, Cylindrospermum, and Phormidium [8]. The entire PKS gene cluster has been sequenced and confirmed to produce anatoxin-a or a variant (homoanatoxina and dihydroanatoxin-a) in Anabaena sp. strain 37, Oscillatoria sp. strain PCC 6506, and Cylindrospermum stagnale PCC 7417 [4, 7-9]. We describe the isolation of a novel anatoxin-a-producing Anabaena from Anderson Lake, Washington State, USA, Anabaena sp. WA102.

Many cyanobacterial genomes remain in draft form (51 according to [10]). Cyanobacteria genomes are often resistant to standard assembly approaches when using Illumina short-insert DNA libraries, due to the fact that they have a large percentage of mobile elements (as much as $11 \%$ of the genome) that repeat throughout the genome [11]. These repeats, and other types of repetitive DNA, are nearly identical in sequence and longer than the insert size of typical DNA sequencing libraries. This causes ambiguous alignment and scaffolding of contigs on either side of the repeat and fragments the genome assembly [12]. While most of the gene content of these genomes properly assembles, reads from mobile element regions usually do not and are omitted from analysis. Structural variation in the genome, such as large deletions or tandem duplications, is also obscured in unfinished genome assemblies. Until recently, the only methods that have spanned repeat regions and produced finished Nostocaceae genomes have been Sanger sequencing and hybrid assembly of 454 and Illumina sequencing libraries that require laborious extra finishing steps. Increasing access to long-read sequencing platforms will circumvent these problems and help to close complex bacterial genomes in a single assembly step [13].

We describe a PacBio sequencing dataset of $8.5 \mathrm{kbp}$ average read length that was used to finish and close the genome of Anabaena sp. WA102. We compare the long-read sequencing results to genome assembly from short-read sequences and describe structural features of potential physiological relevance that are missed by shortread sequencing. We also compare the complete genome of the cultured isolate (Dec 2014) to the population genome of the dominant anatoxin-a-producing Anabaena in Anderson Lake (Jul 2012).

\section{Results}

\section{The Anabaena sp. WA102 culture and genome}

Anabaena sp. WA102 was isolated from a water sample collected during a cyanobacterial bloom in Anderson Lake in Jefferson County, Washington, USA on May 20th, 2013 (Fig. 1a). Anatoxin-a levels in the lake were $12.5 \mu \mathrm{g} / \mathrm{L}$. The non-axenic culture was first established in BG- $11_{0}$ medium, then a single contiguous colony - assumed to be clonal - was isolated from the established culture and serially propagated in BG-110. Colonies from the culture are heterocystous due to lack of nitrogen in the medium and have mean vegetative cell dimensions of 7.1 by $6 \mu \mathrm{m}$ (Fig. 1b). LC-MS/MS analysis showed that the culture produced anatoxin-a, with no detectable homoanatoxin-a nor dihydroanatoxin-a (Additional file 1: Figure S1).

DNA extracted in December 2014 (19 months after culture establishment) was used to construct a library of size-selected fragments (over $8 \mathrm{kbp}$ ) sequenced on four PacBio SMRT cells. A total of 1.13 Gbp with an average read length of $8.5 \mathrm{kbp}$ was sequenced (Table 1 and Additional file 1: Figure S2). Two contigs representing the $5.7 \mathrm{Mbp}$ chromosome and a $76.5 \mathrm{kbp}$ plasmid that make up the complete Anabaena sp. WA102 genome were de novo assembled from the output of two PacBio SMRT cells (Fig. 2a). At an average nucleotide coverage of $72.0 x$, the average Phred quality score for the genome is 81.86 (a $1.5 \times 10^{8}$ probability of an erroneous nucleotide).

The average GC content of the Anabaena sp. WA102 chromosome is $38.4 \%$. There are 5091 predicted genes on the chromosome, including 4667 protein-coding sequences (1824 of which encode hypothetical proteins), 365 pseudogenes, 5 ribosomal RNA operons, and 43 tRNA genes (Table 1). DnaA boxes and a surrounding ATrich region identify a single putative origin of replication from nucleotides 1457-1702. The genome has an unusual GC skew pattern (Fig. 2b) that does not allow for terC site prediction, as also seen with some other cyanobacteria $[14,15]$. rRNA operons are scattered throughout the chromosome, not concentrated near the origin of replication, and in one case oriented against the presumed direction of replication. If Anabaena sp. WA102 is oligoploid like many cyanobacteria [16], then there may be less need to encode highly expressed genes such as the rRNA operons near the origin of replication to increase their copy number or orient them to optimize transcription during replication. The plasmid is $76.5 \mathrm{kbp}$ long $(1.3 \%$ of genome) and has an average GC content of $37.7 \%$. There are 88 genes encoded on the plasmid, including 75 protein coding sequences, the majority of which are hypothetical proteins (57) or pseudogenes (13), and no rRNA or tRNA genes (Table 1).

\section{Comparison of Anabaena sp. WA102 long- and short-read genome assemblies}

DNA from the Anabaena sp. WA102 non-axenic culture was extracted in December 2014 (7 months after culture establishment) and used to construct an Illumina TruSeq metagenome. That library was sequenced as 100nt pairedend reads on the HiSeq 2000 instrument, yielding 3.83 Gbp of total sequence, of which $738 \mathrm{Mbp}$ (19\%) mapped to the closed Anabaena sp. WA102 PacBio genome assembly. A draft Anabaena sp. WA102 genome was extracted from an assembly of this short-read Illumina sequencing 


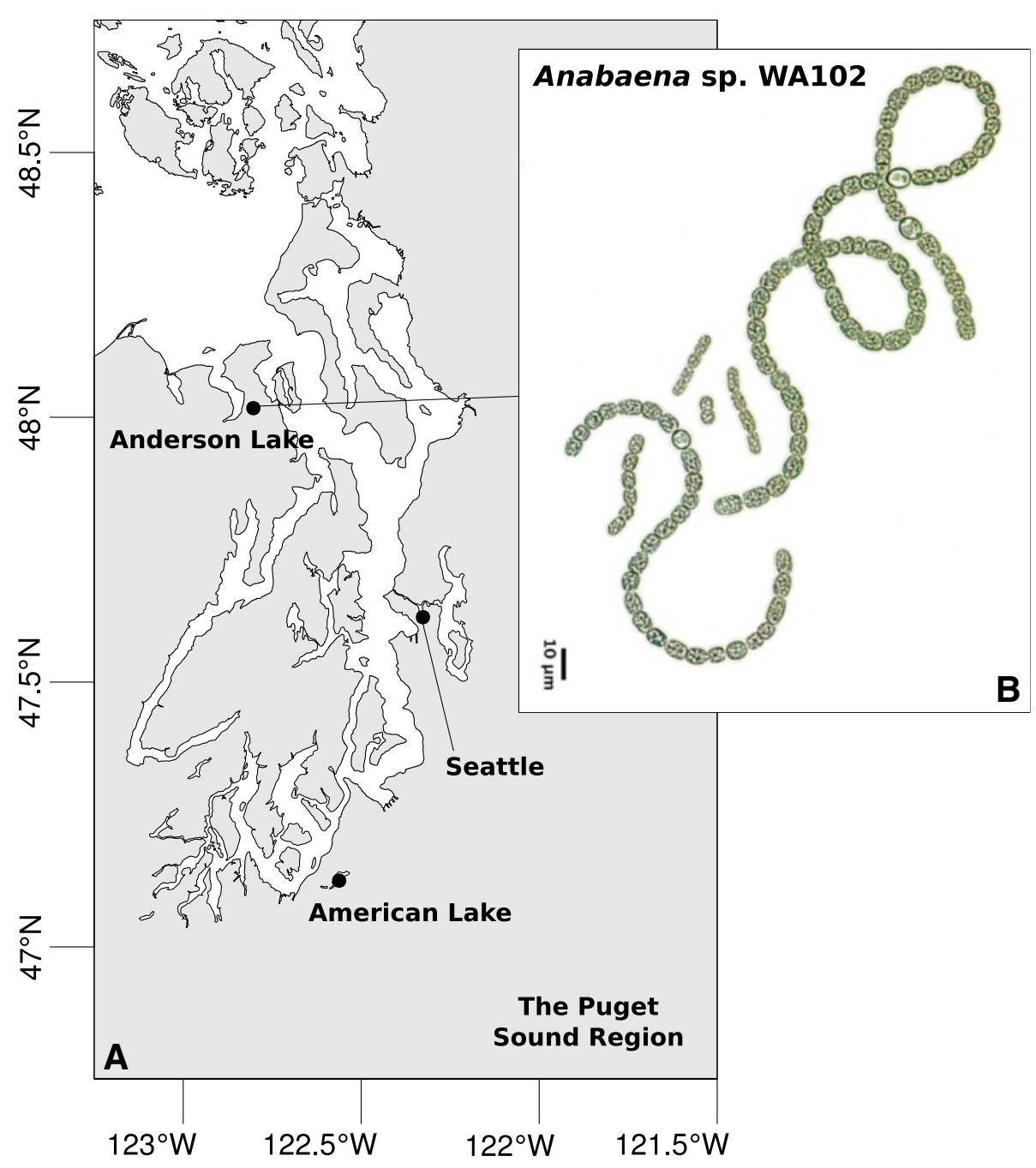

Fig. 1 a A map of the Puget Sound region in Washington State, USA. Anabaena sp. WA102 was isolated from Anderson Lake at 48.0190 N, 237.1963 W on the Olympic Peninsula. b A brightfield micrograph of Anabaena sp. WA102 at 200x magnification. Vegetative cells measure 7.1 by $6 \mu \mathrm{m}$ on average. Colonies are heterocystous because the culture is maintained in nitrogen-free medium (BG-110)

data using the mmgenome package. The draft genome is not complete, but the sum length of contigs in the draft genome is within $1 \%$ of the length of the closed Anabaena sp. WA102 genome. When the draft genome is aligned against an HMM database of essential, universally conserved, bacterial genes from the mmgenome package, 105 essential genes found in other members of the Nostocaceae are also found in the new genomes (compared with 104 essential genes in the closed Anabaena sp. WA102 genome, see Table 2). This suggests that the draft genome is nearly complete and representative of actual gene content. Using blastn, 819 of 820 contigs in the Anabaena sp. WA102 draft genome align to the closed reference genome (e-value $\leq 10^{-30}$ ), further suggesting that the draft genome assembly has little contamination. Some of the contigs in the draft genome overlap when aligned to the closed genome, forming 230 regions of contiguous coverage with 229 gaps that are scattered around the circular genome (Fig. 2a).

The gap regions sum to $34,166 \mathrm{bp}$ ( $0.6 \%$ of the reference genome), containing 97 genes. Over half of these (56 genes) have more than one copy in the genome, including 26 genes from a single cluster of transposases. Many single-copy hypothetical genes that coincide with gaps have low complexity regions. Most gaps (green tiles on Fig. 2a) coincide with long repeat regions in the genome, whose multiple copies are connected by green arcs (Fig. 2a). The repeat regions include the five rRNA operons, genes encoding transposons and homing endonucleases, and other repeat regions discussed in more detail below. In some cases gaps coincide with GC-rich regions. These results agree with previous observations of gaps in 
Table 1 Summary statistics of sequencing data and binned Anabaena genomes

\begin{tabular}{|c|c|c|c|c|c|c|c|c|c|}
\hline \multirow[t]{2}{*}{ Sample } & \multirow{2}{*}{$\begin{array}{l}\text { Sample } \\
\text { date }\end{array}$} & \multirow{2}{*}{$\begin{array}{l}\text { Library prep/ } \\
\text { Seq platform }\end{array}$} & \multirow{2}{*}{$\begin{array}{l}\text { Seq } \\
\text { output } \\
\text { (Gbp) }\end{array}$} & \multicolumn{6}{|c|}{ Anabaena sp. genome } \\
\hline & & & & N50 & $\begin{array}{l}\text { Mean } \\
\text { cov. }\end{array}$ & $\begin{array}{l}\text { No. } \\
\text { contigs }\end{array}$ & $\begin{array}{l}\text { Max } \\
\text { contig } \\
\text { (nt) }\end{array}$ & $\begin{array}{l}\text { Total } \\
\text { length } \\
\text { (nt) }\end{array}$ & $\begin{array}{l}\text { Unique } \\
\text { core } \\
\text { genes }\end{array}$ \\
\hline $\begin{array}{l}\text { Anabaena } \\
\text { sp. WA102 } \\
\text { culture } \\
\text { est. from }\end{array}$ & $\begin{array}{l}\text { Dec } \\
2014 \\
\text { Dec }\end{array}$ & $\begin{array}{l}\text { Blue } \\
\text { Pippin/ } \\
\text { PacBio } \\
\text { TruSeg/ }\end{array}$ & 1.13 & $5,715,573$ & $50 x$ & $3^{*}$ & $5,705,437$ & $5,807,452$ & 104 \\
\hline $\begin{array}{l}\text { est. from } \\
\text { Anderson } \\
\text { Lake } \\
\text { May } 2013\end{array}$ & $\begin{array}{l}\text { Dec } \\
2013\end{array}$ & $\begin{array}{l}\text { TruSeq/ } \\
\text { HiSeq2000 } \\
\text { 100bp PE }\end{array}$ & 3.83 & 15,892 & $129 x$ & 819 & 66,878 & $5,698,213$ & 105 \\
\hline WA25 & $\begin{array}{l}\text { July } \\
\text { 2012/ } \\
\text { Anderson } \\
\text { Lake }\end{array}$ & $\begin{array}{l}\text { TruSeq/ } \\
\text { HiSeq2000 } \\
\text { 100bp PE }\end{array}$ & 30.1 & $\begin{array}{l}\text { Shotgun } \\
\text { metagenome } \\
\text { of surface } \\
\text { lake water }\end{array}$ & - & - & - & - & - \\
\hline $\begin{array}{l}\text { Anabaena } \\
\text { sp. AL93 } \\
\text { culture } \\
\text { est. from } \\
\text { American } \\
\text { Lake } \\
1993\end{array}$ & $\begin{array}{l}\text { Jan } \\
2013\end{array}$ & $\begin{array}{l}\text { Nextera/ } \\
\text { MiSeq } \\
\text { 250bp PE }\end{array}$ & 1.36 & 46,264 & $149 x$ & 314 & 133,848 & $5,757,055$ & 105 \\
\hline
\end{tabular}

PE indicates paired-end reads. *Three contigs include the chromosome, plasmid, and the contig representing the insertion variant with the xseA gene

Illumina assemblies due to long repeat regions and regions of low nucleotide complexity [12]. The large number of contigs generated from the short-read Illumina sequences emphasizes the prevalence of long repetitive elements in the Anabaena sp. WA102 genome and the value of long-read sequencing technologies in producing finished genomes. This is further demonstrated by observations of tandem repeats in the long-read assembly, observation of structural variants in the population, analysis of genome synteny with another closely related Anabaena genome, and a full count of mobile elements within the genome (described below).

\section{The Anabaena sp. AL93 culture and genome}

Anabaena sp. AL93 is an anatoxin-a producing strain isolated in non-axenic culture from a toxic bloom in American Lake, Washington in 1993 (MA Crayton, personal communication). It provides local geographical context for Anabaena sp. WA102, since American Lake is only $100 \mathrm{~km}$ from Anderson Lake. It also provides some evolutionary context as a close relative of Anabaena sp. WA102 (see phylogeny below). The genome was sequenced with $1.36 \mathrm{Gbp}$ of Illumina MiSeq 250-bp paired-end reads. Contigs representing $5.7 \mathrm{Mbp}$ of the Anabaena sp. AL93 draft genome were binned using the mmgenome package to yield a nearly complete genome with 105 essential genes according to the database in the mmgenome package.
Phylogenomic relationship between Anabaena sp. WA102, AL93, and other fully sequenced Nostocaceae

The closed genome from Anabaena sp. WA102 and the draft genome from Anabaena sp. AL93 can be placed phylogenetically among draft and full genomes from members of the Nostocaceae. The ancestral relationship of eleven genomes from the Nostocaceae was hypothesized with a phylogenomic tree based on 1408 clusters of unique orthologs from each genome (Fig. 3). Unanimity among 1000 tree constructions yielded $100 \%$ bootstrap support for every internal node. The tree was rooted at Nostoc sp. PCC 7107, according to [10]. Anabaena sp. WA102 and Anabaena sp. AL93 are most closely related to each other. They form a distinct clade with Anabaena sp. 90, a microcystin toxin-producing strain from Finland [15].

\section{Comparing gene content and metabolic capabilities of Anabaena sp. WA102 and AL93 with other Nostocaceae genomes}

The gene contents of Anabaena sp. WA102 and closely related Nostocaceae genomes were also assigned to metabolic pathways using the KEGG ortholog database. All genes necessary for nitrogen fixation (nifDKH) were found throughout these genomes. Figure 4 highlights metabolic pathways with differential representation in Anabaena sp. WA102 and its relatives. Differences in sulfur metabolism are evident among the genomes. The $s s u$ operon, which is involved in transport and metabolism of 


$$
\text { ? }
$$


Table 2 Summary of Anabaena sp. WA102 genome (Genbank:CP011456-7) annotation according to the Prokka script and NCBI Prokaryotic Annotation Pipeline

\begin{tabular}{|c|c|c|c|c|}
\hline \multicolumn{5}{|c|}{ Chromosome } \\
\hline Category & Element & $N C B I$ & Prokka & Manual \\
\hline \multirow[t]{5}{*}{ Protein-coding genes: } & Total & 4667 & 5175 & NA \\
\hline & Hypothetical proteins & 1824 & 2187 & NA \\
\hline & Transposases & 79 & 82 & 130 \\
\hline & Homing endonucleases & 7 & 30 & 30 \\
\hline & Histidine kinases & 25 & 26 & NA \\
\hline \multirow[t]{3}{*}{ RNA genes: } & rRNA operons & 5 & 5 & NA \\
\hline & tRNAs & 43 & 44 & NA \\
\hline & Riboswitches & 2 & NA & NA \\
\hline \multirow[t]{6}{*}{ Pseudogenes: } & Total & 365 & NA & NA \\
\hline & Hypothetical proteins & 186 & NA & NA \\
\hline & Transposases & 29 & NA & NA \\
\hline & Homing endonucleases & 6 & NA & NA \\
\hline & Histidine kinases & 1 & NA & NA \\
\hline & Plasmid & & & \\
\hline \multirow[t]{4}{*}{ Protein-coding genes: } & Total & 75 & 96 & NA \\
\hline & Hypothetical proteins & 57 & 66 & NA \\
\hline & Transposases & 3 & 2 & NA \\
\hline & Homing endonucleases & 0 & 0 & NA \\
\hline \multirow[t]{4}{*}{ Pseudogenes: } & Total & 13 & NA & NA \\
\hline & Hypothetical proteins & 10 & NA & NA \\
\hline & Transposases & 0 & NA & NA \\
\hline & Homing endonucleases & 1 & NA & NA \\
\hline
\end{tabular}

organic sulfur compounds [17], was intact in Anabaena sp. WA102. It was absent or incomplete in 6 of 11 Nostocaceae, including Anabaena sp. 90. ssuABCDE and tauD (taurine metabolism) are in the same gene cluster in Anabaena sp. WA102 and are likely co-regulated. Anabaena sp. WA102 also possesses the fhuBC genes, which encode two parts of the ferric hydroxamate $A B C$ transporter. The presence of these genes suggest that Anabaena sp. WA102 is well equipped to import organic sulfur compounds and iron from the environment. This may provide a competitive advantage in providing the iron-sulfur clusters that are necessary for nitrogen fixation in niches with low sulfate availability.

Other genes present in Anabaena sp. WA102 but not in Anabaena sp. 90 or other Nostocaceae (Fig. 4) may also provide competitive advantage under certain conditions. $b t u B$ is necessary for vitamin B12 uptake from the environment [18]. The urt $A B C D E$ cluster allows uptake and metabolism of nitrogen-rich urea [19]. cyd $A B$ encode the cytochrome $b d$-type oxidase, which has been shown to be necessary for Nostoc sp. PCC 7120 survival under nitrogen-limited conditions and is hypothesized to scavenge oxygen in heterocysts to prevent oxidation of nitrogenase [20]. The presence of pixGHIJL genes, which encode a phototactic system, suggests that Anabaena sp. WA102 is positively phototactic and likely motile [21]. In support of this hypothesis, Anabaena sp. WA102 encodes a twitching-motility pilus gene pilT, and a pilus assembly gene pilC (loci AA650_16975 and 16980). Gas vesicle genes present in two clusters (loci AA650_0781 to 07850 and AA650_07865 to 07870) support mobility through buoyancy control [22].

A number of metabolic genes are absent from Anabaena sp. WA102, but present in Anabaena sp. 90 or other Nostocaceae. pecABCEF, the genes responsible for phycoerythrocyanin synthesis [23]), are absent from Anabaena sp. WA102 but present in its close relative Anabaena sp. AL93 [24]. Phycoerythrocyanin is a photosynthetic pigment that absorbs light maximally at 575nm (green light) and confers a competitive advantage in coastal and freshwater environments where phytoplankton and turbid waters absorb much of the red light that is maximally absorbed by the ubiquitous phycocyanin pigment [23]. These two strains can be distinguished by their pigments, a critical element in niche adaptation. Both strains encode genes to synthesize the phycobilins phycocyanin and allophycocyanin, but only Anabaena sp. AL93 encodes the genes for phycoerythrocyanin synthesis. The absence of a phycoerythrocyanin operon suggests that Anabaena sp. WA102 would not compete well in shade from other photosynthetic organisms or deeper and murkier water because it cannot efficiently absorb green light. Rather, it may avoid shade or deeper water by positive phototaxis to the lake surface driven by gas vesicle buoyancy. The psbJLM components of the photosystem II apparatus are intermittently distributed throughout the Nostocaceae in this study but are completely absent from Anabaena sp. WA102. Different combinations of light harvesting genes in each genome, without a phylogenetic pattern, suggest that they are selected for under different light conditions and perhaps horizontally transferred.

\section{Capacity for synthesis of anatoxin-a and other secondary metabolites}

Cyanobacteria produce many secondary metabolites, including products of nonribosomal peptide synthetase (NRPS) and polyketide synthase (PKS) genes. Much concern about freshwater cyanobacterial blooms stems from their production of toxic secondary metabolites. Fourteen gene clusters in the Anabaena sp. WA102 genome encode putative secondary metabolite synthesis proteins (Fig. 2a and Additional file 1: Table S1). Anatoxin-a is made by proteins encoded in cluster eleven located 


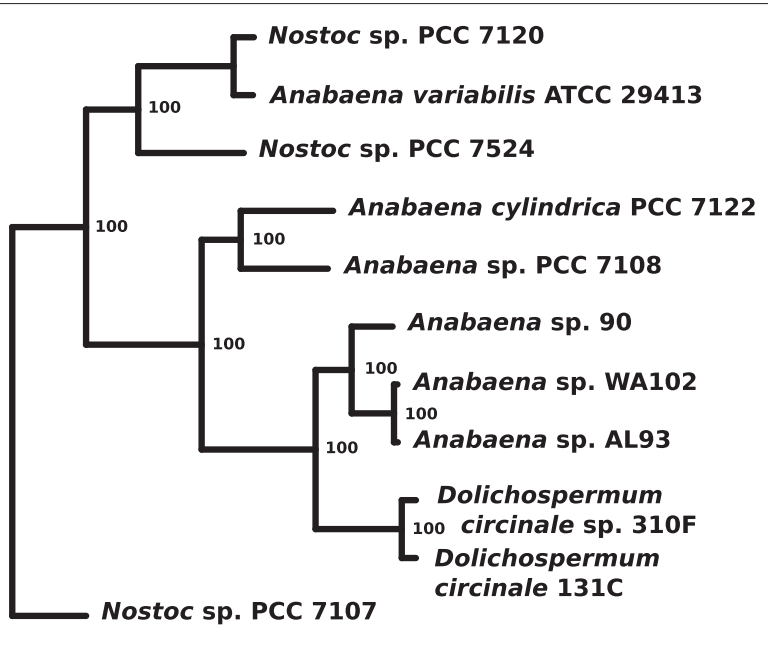

Fig. 3 A phylogenomic tree constructed from amino-acid alignments of single-copy orthologs present in all genomes of some of the fully sequenced members of the Nostocaceae

between nucleotides 4,362,415 and 4,392,159, confirming that Anabaena sp. WA102 indeed is able to produce anatoxin-a, as detected by LC-MS/MS (Additional file 1: Figure $\mathrm{S} 1$ ). The anaA-G genes in this $30 \mathrm{kbp}$ cluster are syntenous with homologs in Anabaena sp. 37 and Anabaena sp. AL93 (Fig. 5). However, genes anaA, anaI, and $a n a J$ are rearranged between the Anabaena anatoxina clusters and the Oscillatoria and Cylindrospermum clusters [7].

Comparing ana clusters between Anabaena sp. WA102, Anabaena sp. AL93, Anabaena sp. 37, Cylindrospermum stagnale sp. PCC 7417, and Oscillatoria sp. PCC
6506 showed differences in the anaG gene (Fig. 5 and Additional file 1: Figure S3). The AnaG protein plays a key role in determining the anatoxin variant produced [7]. AnaG adds an acetyl group and either one or two methyl groups to the bicyclic thioester precursor, forming either anatoxin-a or homoanatoxin-a, respectively. Oscillatoria sp. PCC 6506, which produces $99 \%$ homoanatoxin-a and $1 \%$ anatoxin-a, possesses the largest methyltransferase domain in AnaG. The smaller AnaG methyltransferase domain in Anabaena sp. 37, a producer of anatoxin-a [8], is evidently not involved in homoanatoxin-a synthesis. The AnaG methyltransferase domain is missing entirely in

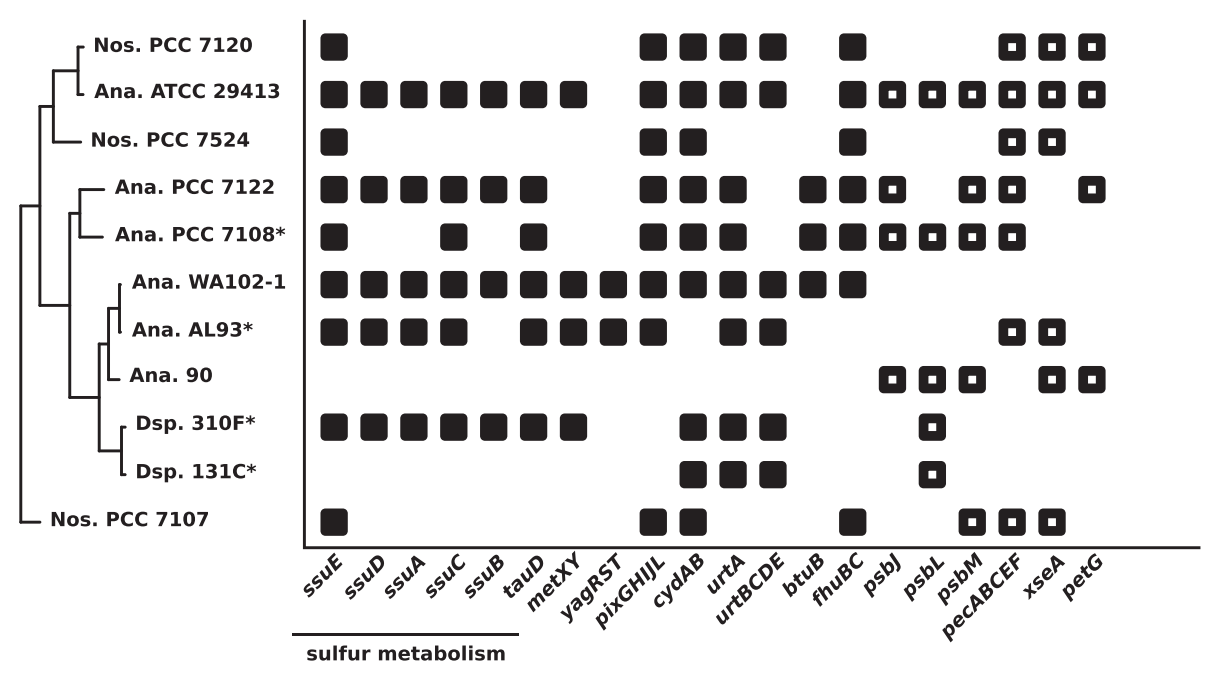

Fig. 4 KEGG orthologs (KO) differentially represented among the compared Nostocaceae genomes. All proteins from each Nostocaceae genome were mapped to the online KO database. Orthologs with significant differences among the genomes were highlighted in the above table for comparison. Nostocaceae genomes are arranged according to the phylogenomic tree for easy comparison. The Anabaena sp. WA102 genome encodes a sulfur metabolism cluster absent or incomplete in 6 out of 11 Nostocaceae genomes 


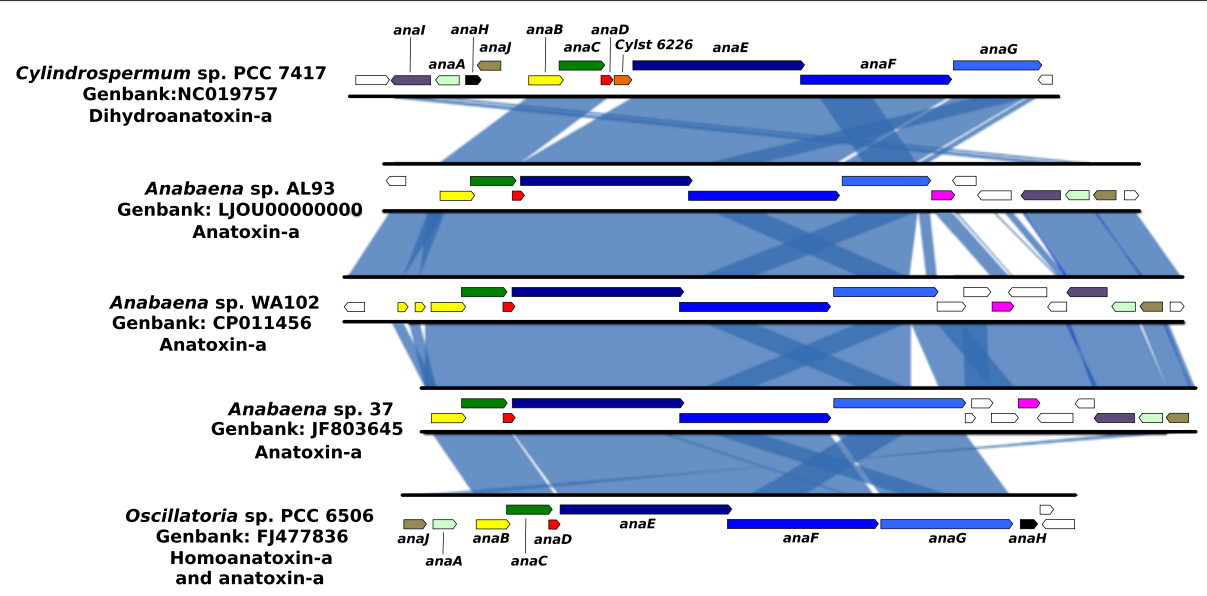

Fig. 5 Nucleotide alignment of anatoxin-a clusters from Cyanobacteria. anaA-G and anal are all conserved in Anabaena sp. WA102 and Anabaena sp. AL93, though anaH is missing from both. The $5^{\prime}$ region of $a n a B$ and upstream promoter region is triplicated in Anabaena sp. WA102. The anatoxin-a cluster from Anabaena sp. WA102 is most similar to that from Anabaena sp. 37. The three Anabaena strains share a gene of unknown function downstream of anaG (colored pink). The anaG genes differ in size, correlated with different variants of anatoxin-a. Shorter variants of AnaG omit or truncate a putative methyl transferase domain. The anaF and anaG genes share a region of $86 \%$ nucleotide identity that is likely a homologous protein domain. Anabaena sp. WA102 and AL93 encode two of the shortest anaG genes and produce anatoxin-a, Cylindrospermum sp. PCC 7417 produces dihydroanatoxin-a (likely due to the unique gene Cylst 6226), and Oscillatoria sp. PCC 6506 primarily produces homoanatoxin-a

Anabaena sp. WA102 and Anabaena sp. AL93, which are also producers of anatoxin-a (Fig. 5 and Additional file 1: Figure S3). In Cylindrospermum sp. PCC 7417, which produces dihydroanatoxin-a, AnaG lacks the methyltransferase domain as well as the phosphopantetheine transferase domain on the extreme C-terminus (Additional file 1: Figure S3). In the same strain, an oxidoreductase gene, Cylst6226, not present in the other ana clusters, is present (Fig. 5) and implicated in dihydroanatoxin-a synthesis [7]. Note that annotation of genes anaH-J differs between [7] and [4]; we have chosen to follow [7].

The anatoxin-a synthetase gene cluster from Anabaena sp. AL93 revealed an organization most similar to that of Anabaena sp. WA102, although the AL93 AnaG gene is shorter in the C-terminal region. There are also differences in genes situated between anaG and anaI, which include genes not thought to be involved in anatoxin synthesis. Notably, all clusters (not shown for Oscillatoria sp. PCC 6506 in Fig. 5, but referred to in [7]), share a MATE efflux pump homolog (anaI). MATE efflux pumps encoded within the saxitoxin gene cluster are known to export saxitoxin, another toxic secondary metabolite, from the producing cell [25]. They may play a similar role with anatoxin-a.

\section{Lack of synteny with Anabaena sp. 90}

Among the completely sequenced Anabaena genomes, Anabaena sp. WA102 is most closely related to Anabaena sp. 90, sharing an average nucleotide identity (ANI) of 91.5 $\%$ and 2331 gene homologs. Despite this relatively close relationship, there are major differences in overall genome architecture. Whereas the Anabaena sp. 90 genome has two chromosomes of 4.33 and $0.82 \mathrm{Mbp}$, Anabaena sp. WA102 has a single chromosome. Local nucleotide alignment showed that there is little long-range synteny between the two Anabaena genomes (Additional file 1: Figure S4).

Novichkov et al. illustrated common paradigms of synteny between genomes within a genus using dotplots [26]. Aligning genomes between species of Pseudomonas yielded long stretches of synteny, but aligning genomes between species of Streptococcus showed no synteny. Those dotplots are recreated and shown beside the dotplot for Anabaena sp. 90 and WA102 (Fig. 6). Orthologs from each pair of aligned genomes were aligned by BLASTP, showing that average amino acid identity between orthologs of the Anabaena genomes was the highest (Fig. 6). The dotplot of the Anabaena genomes is very fragmented, although these genomes are relatively closely related. The distinct X-shape to dotplots of Pseudomonas and Streptococcus genomes indicate chromosomal inversions around the origin of replication [27]. This pattern is missing in the dotplot of Anabaena genomes, indicating the infrequency or absence of these inversions. Figure 6 indicates that the Anabaena genomes have experienced a relatively faster rate of recombination versus point mutation. This is not uncommon among bacterial genomes but varies among different taxa [28]. Length distributions of the local colinear blocks (LCB's) from alignments calculated by Mauve (Additional file 1: Figure S5) support the general disruption of gene order between Anabaena sp. WA102 and 90. The largest local colinear 


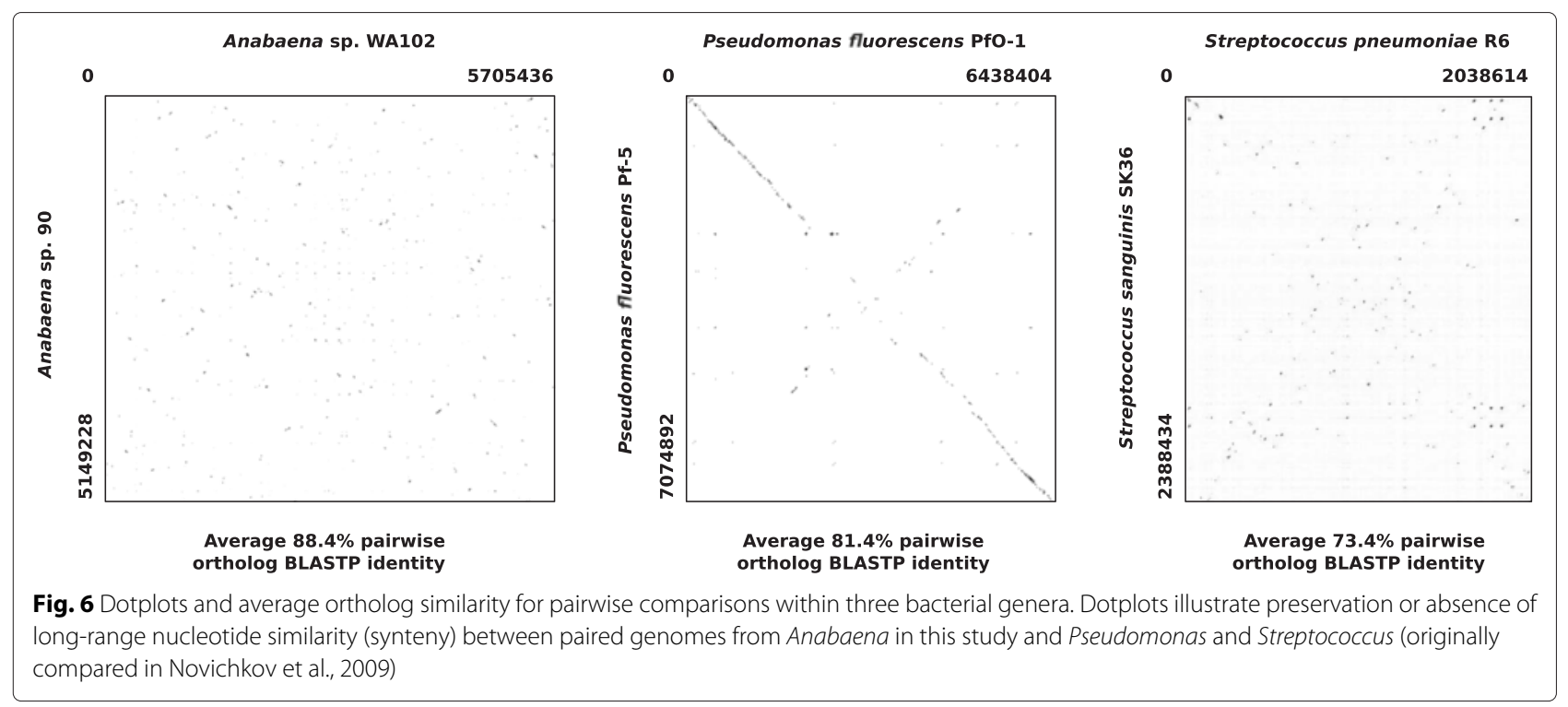

blocks (Additional file 1: Table S2) encompass biosynthetic gene clusters and a cryptic prophage discussed below. The LCBs are not clearly bounded by either repeat sequences or mobile elements, which does not lend a clear explanation for their rearrangement between the two bacteria.

In addition to long-range shuffling, we also detected local rearrangement of genes within clusters. For instance, an LCB at nucleotides 1,992,912-2,007,469 that includes thirteen genes in Anabaena sp. WA102 corresponds to the region between nucleotides $3,575,881-3,591,878$ in Anabaena sp. 90 that includes fourteen genes (Additional file 1: Figure S6). Genes in this syntenous region are putatively involved in complex carbohydrate biosynthesis and export (being mostly glycosyltransferases and including an $\mathrm{ABC}$ transporter). Of these, two glycosyltransferases, an acyltransferase, and a hypothetical protein are unique to Anabaena sp. WA102 and six glycosyltransferases are unique to Anabaena sp. 90. The remaining nine genes in Anabaena sp. WA102 and eight genes in Anabaena sp. 90 are homologous or share homologous domains. Two transposases are responsible for interrupting just one portion of synteny in this region, leaving 4 breaks in synteny unexplained. This suggests that recombination interrupts synteny even in otherwise conserved gene clusters, though the mechanism for recombination is not always clear.

\section{The mobilome}

One hundred eight transposases (79 intact and 29 pseudogenes) were automatically annotated by the NCBI pipeline, constituting $2 \%$ of the genome. Manual annotation with the aid of the IS Finder database [29] increased the number of intact and fragmented transposases to 130.
In addition to transposases, $30 \mathrm{HNH}$ homing endonuclease reverse transcriptases are encoded in the Anabaena sp. WA102 genome, bringing the total number of intact and degenerate mobile elements to 160 . Phylogenetic relationships between insertion sequences show that two groups of closely related IS4-family insertion sequences predominate (20 in the IS10-like group and 25 in the IS4Sa-like group) among a wider representation of IS families (Fig. 7). Aligning nucleotide sequences adjacent to each side of the coding sequence of these insertion sequences revealed the unique inverted repeat sequence for each group: ATTCAACAYTTCTG for the IS10like group, and CCGCCTTGTCACCCGTTAAG for the IS4Sa-like group. These two groups of transposases catalyze their transposition via three acidic residues in their active site: two aspartates and a glutamate, and transpose in a cut-and-paste fashion (non-replicative) [30].

Other common mobile elements found in bacterial genomes are prophages, cryptic prophages, and phagelike elements such as gene transfer agents (GTAs). No signature phage regions were detected with the PHAST phage-detection webserver. The IslandViewer 3 webserver, which detects genomic islands, highlighted an 18 kbp region between nucleotides 1,179,961 and 1,198,734. This region is also contained in the largest local colinear block (LCB) calculated by Mauve between Anabaena sp. WA102 and Anabaena sp. 90 (Additional file 1: Table S2). Within the LCB, there is a $19 \mathrm{kbp}$ insertion in Anabaena sp. WA102 relative to Anabaena sp. 90. The LCB boundaries likely denote the exact boundaries of a cryptic prophage: nucleotides 1,179,211-1,198,554. This region contains a putative phage terminase large subunit that was automatically annotated by Prokka and confirmed with $100 \%$ confidence by Phyre 2 structure-guided annotation. 


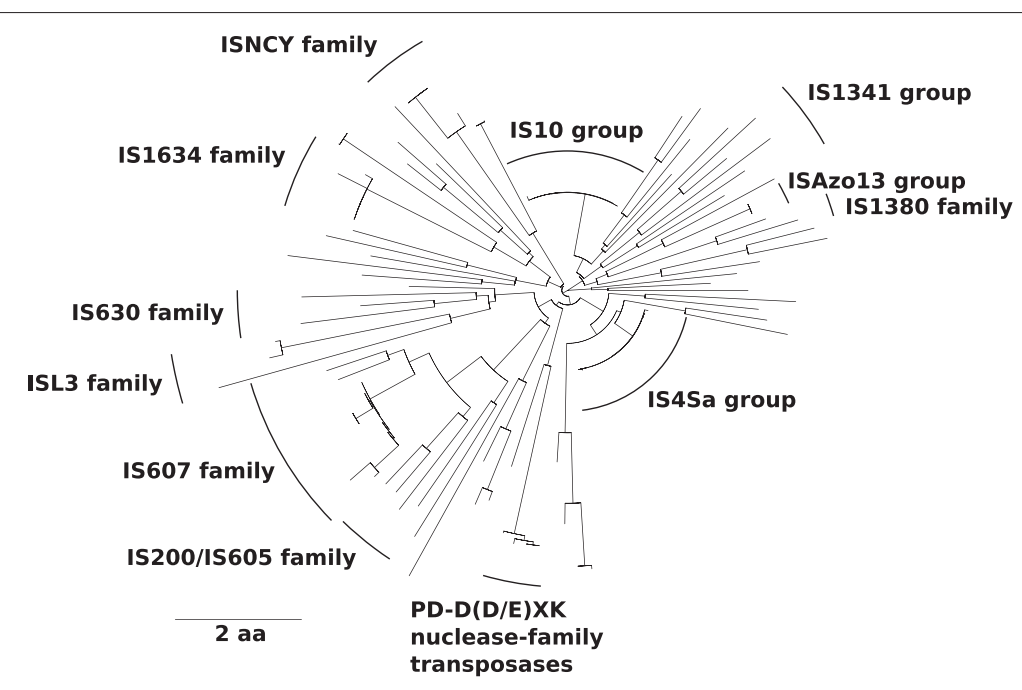

Fig. 7 Phylogenetic tree of transposase protein sequences encoded in the Anabaena sp. WA102 genome. The phylogenetic relationship between 130 annotated transposase protein sequences is sketched out in the tree. Two large clades of closely related transposases dominate the tree. The IS4Sa clade includes 25 transposases and the IS10 clade includes 20 transposases, which both belong to the larger IS4 transposase family. These transposases have a DDE-type active site that facilitates cut-and-paste transposition. The IS4Sa clade has an identical terminal direct repeat sequence: CCGCCTTGTCACCCGTTAAG. The IS10 clade has the terminal direct repeat sequence: ATTCAACAYTTCTG

The terminase large subunit is a component of a DNA packaging protein unique to Caudovirales. Within this region also lie 21 hypothetical proteins, one IS-4 family transposase, one pseudogene, and one integrase. The large proportion of hypothetical proteins is consistent with a phage origin. The integrase lies 134 nucleotides downstream of a methionine tRNA, which may have served as an integration site (attB) of the prophage. The GC content in the region is $32.9 \%$, lower than the genome average of $37.7 \%$ and consistent with a horizontally transferred region that has a distinct nucleotide composition. The small size of the region, lack of other identifiable phage proteins such as capsid or tail structure proteins, and the insertion of a transposon common to the bacterial genome suggest that this region is a partly degraded cryptic prophage. Several other phage integrases were automatically annotated, but these integrases are often functionally mislabeled. Alternatively, they may be sitespecific integrases native to or co-opted by the bacterial genome for functions other than prophage integration and excision. These alternative functions are likely, considering the absence of other readily identifiable phage genes near these integrases.

Besides transposons and phage-like elements, a single plasmid was identified, rounding out the mobile element complement of Anabaena sp. WA102. The plasmid was identified as a $92 \mathrm{kbp}$ contig assembled from PacBio reads. Fifteen kbp of nucleotide sequence from each end of the contig aligned with $99 \%$ similarity (overlapped with lower quality sequence at the extremities) and was excised from the final plasmid. The trimmed plasmid sequence is 77 $\mathrm{kbp}$ long, with a $37.2 \%$ average GC content. The 88 genes on the plasmid include 75 intact and 13 pseudogenes. A par $A B$ operon on the plasmid suggests that it is a lowcopy plasmid (confirmed by an average read coverage less than that of the chromosome) with a well described partitioning mechanism [31]. The parAB operon and surrounding nucleotide sequence bears at least $86 \%$ similarity to the par $A B$ operon and its surrounding sequence on the chromosome (Additional file 1: Figure S7). Interestingly, the plasmid carries at least part of a non-ribosomal peptide synthase (NRPS) cluster. One protein within the cluster shows significant similarity to AdpD from the anabaenopeptilide cluster in Anabaena sp. 90 (BLASTP e-value $=6.9 \times 10^{-121}$ ). The other three biosynthetic proteins in the cluster show similarity to a malonyl CoA-acyl carrier protein transacylase, a $\beta$-ketoacyl synthase, and a short-chain dehydrogenase. Plasmid-borne NRPS clusters are not uncommon. A recent comprehensive survey of NRPS and polyketide synthase (PKS) clusters in all bacterial genomic data deposited at the National Center for Biotechnology Information (NCBI) revealed that $10 \%$ of NRPS/PKS clusters in Cyanobacteria are located on plasmids [32]. Importantly, the plasmid encodes four putative site-specific integrases, which may facilitate integration into a bacterial chromosome. Coupled with nucleotide similarity between the plasmid and the chromosome, where site-specific integrases can also be found, this indicates that the region of plasmid similarity on the chromosome may be considered a genomic island. 


\section{Relationship between the Anabaena sp. WA102 genome and the Anderson Lake metagenome}

To relate the Anabaena sp. WA102 genome to a bloom in Anderson Lake, the WA25 metagenome was sampled from Anderson Lake on July 7th, 2012 (unpublished data). The sample was taken near the peak of a cyanobacterial bloom, when the anatoxin-a level was $187 \mu \mathrm{g} / \mathrm{L}$ (https:// www.nwtoxicalgae.org/Data.aspx). The metagenome contains a genome from a strain of Anabaena sp. WA102 that is nearly identical to the culture and is likely an ancestor from 10 months before the culture strain was isolated and 2.5 years before it was sequenced. Reads from the July 2012 metagenome short-read Illumina), the December 2013 culture (short-read Illumina), and the December 2014 culture (long-read PacBio) were mapped to the closed reference genome to track changes in the genome over time.

\section{A recent deletion event in the Anabaena sp. WA102 genome}

The length of the PacBio reads not only allowed us to close the Anabaena sp. WA102 genome but also revealed structural variation in the population. The $21 \mathrm{kbp}$ segment between nucleotides 4,790,517 and 4,812,024 was also present (99\% similarity) on a $25 \mathrm{kbp}$ contig in the PacBio assembly, reflecting the existence of a $4 \mathrm{kbp}$ indel variant within the genomes of the Anabaena sp. WA 102 culture population (Fig. 8). Mapping reads from the Anabaena sp. WA102 PacBio dataset showed that the contig had an average coverage of $25 \mathrm{x}$, approximately one-third of the average coverage of the chromosome $(73 \mathrm{x})$, and that the deletion actually lies between nucleotides 4,800,950 and $4,804,900$. This suggests that the deletion is present in two-thirds of the Anabaena sp. WA102 culture population. The indel appears to be a deletion that arose after December 2013, since the longer sequence is predominant in sequencing reads from both the July 2012 metagenome and the December 2013 culture (Fig. 8). An XseA homolog (the large subunit of exonuclease VII) and two hypothetical gene products are deleted in the variant. In well characterized Escherichia coli xseA mutants, there is an increased recombination phenotype [33], suggesting the same may be true for two-thirds of the Anabaena sp. WA102 culture population.

\section{Tandem repeat of the anatoxin-a anaBCD promoter region} Intriguingly, the anatoxin-a synthase region in the PacBio assembly of Anabaena sp. WA102 showed that the first $173 \mathrm{bp}$ of the $a n a B$ gene and $398 \mathrm{bp}$ upstream of the gene had been triplicated (Figs. 5 and 9). This is in contrast with the genome of Anabaena sp. AL93, which does not have a triplication of the $a n a B$ promoter region. The 398 nucleotides upstream of $a n a B$ include four highscoring putative transcriptional regulation binding sites and promoters, identified in silico using Virtual Footprint and the PRODORIC database of position weight matrices for bacterial transcriptional regulation binding sites and promoters [34]. Assembling Illumina reads from the Anabaena sp. WA102 culture with IDBA v1.1.1 and PriceTI fails to correctly resolve the tandemly triplicated promoter region (Fig. 9a). To determine when this triplication arose, reads from the July 2012, Dec 2013, and Dec 2014 sequencing runs were mapped to the triplicated region (Fig. 9b). Illumina reads from the Anderson

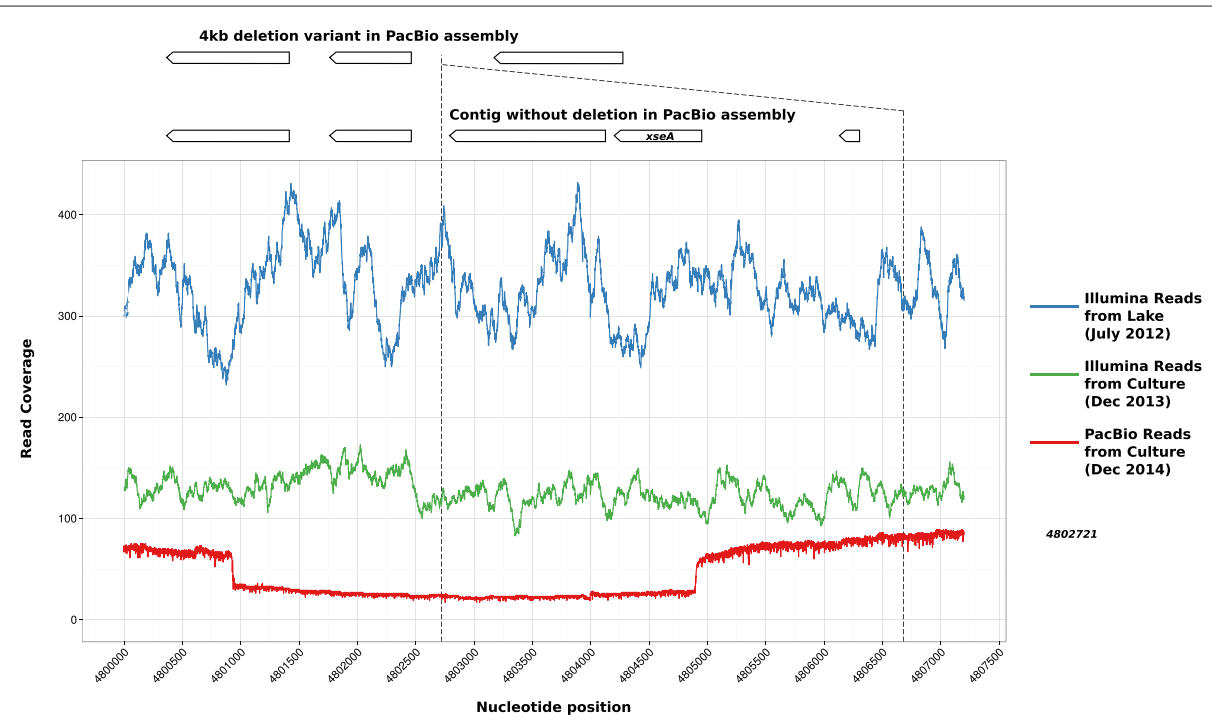

Fig. 8 A deletion mutation was detected in the PacBio long-read assembly of the Anabaena sp. WA102 culture. Mapping reads to the indel region showed that the deletion occurred between nucleotides 4,800,950 and 4,804,900. The deletion arose after December 2013 and expanded through the population to roughly two-thirds of the culture population by December 2014 


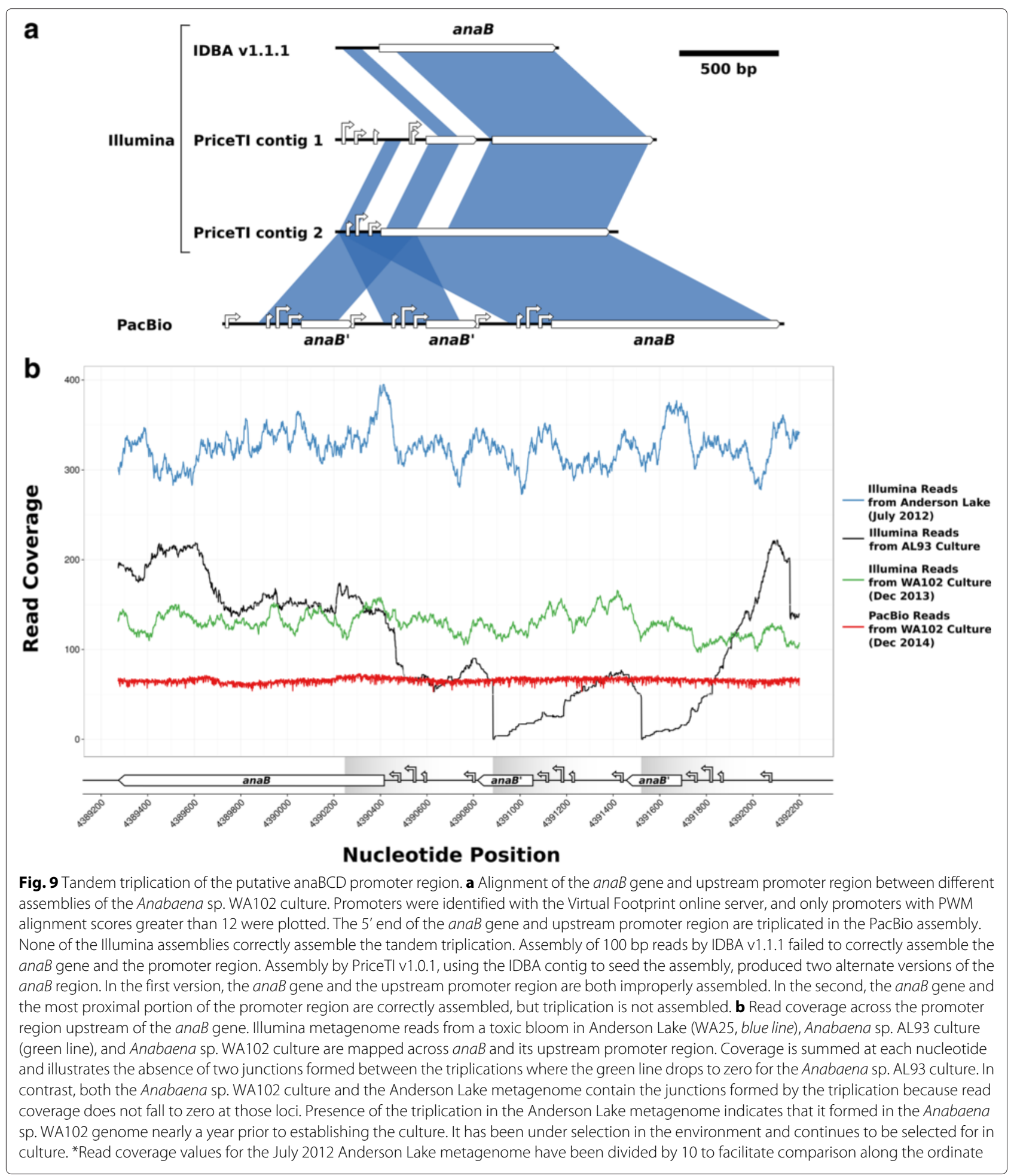

Lake metagenome and the Anabaena sp. WA102 culture mapped across the two unique junctions formed by the triple tandem repeats, confirming its presence as early as 2012 in Anderson Lake and also in the culture sequenced in December of 2013. In contrast, none of the reads from the Anabaena sp. AL93 culture mapped across the unique junctions formed by the tandem repeats (indicated by arrows in Fig. 9b). This triplication is unique to Anabaena sp. WA102 among all known anatoxin-a cluster sequences and has been stable for at least 2.5 years, in both Anderson 
Lake and under culture conditions. Toxin production has been measured in the culture (Additional file 1: Figure $\mathrm{S} 1$ ), so the tandem repeat is not interrupting transcription of the anaBCD operon. Instead, triplication of the putative promoter region may increase transcription of the operon.

\section{Discussion and conclusions}

The recently cultured toxic isolate, Anabaena sp. WA102, closely reflects the parent population in Anderson Lake

Anabaena sp. WA102 is a novel anatoxin-a-producing member of the Nostocaceae isolated from Anderson Lake on the Olympic Peninsula in Washington in 2013. It is in stable non-axenic culture. The Anabaena sp. WA102 genome is unique among sequenced Anabaena genomes because it was sequenced within seven months of isolation. Other Anabaena strains have been in culture for several decades prior to whole genome sequencing and changes in a strain's genome can accumulate over such long periods. Sequencing a strain soon after isolation increases the relevance of the sequenced genome to the environment from which it was isolated and provides a reference point for later studies of the strain's genome.

Anabaena sp. WA102 produces anatoxin-a in culture (Additional file 1: Figure S1). The toxin is produced by NRPS and PKS enzymes encoded by the anaA-J gene cluster. A triple tandem repeat of the $a n a B$ putative promoter region in the cultured isolate (Fig. 9a) is present in a nearly identical strain in the environment (July 2012 Anderson Lake sample, WA25 in Table 2), which suggests that it originates from and is relevant to the lake environment. Tandem repeats of genes and promoters commonly arise in bacterial genomes but are unstable and can collapse through homologous recombination or strand slippage at high frequency, unless the repeat is under selection $[35,36]$. Thus, tandem repeats have been hypothesized to act as a crude selection-regulated response to environmental change [37-39]. Additionally, tandem repeats provide redundancy that drives the innovation, amplification, divergence (IAD) cycle that generates genetic novelty [40]. Tandemly repeated promoters, in particular, allow for promoter regions to generate or acquire new regulatory binding sites that can change the expression pattern of an operon [41]. Further study of this tandem repeat may be fruitful for several reasons. Most noteworthy is that these tandem repeats are $617 \mathrm{nt}$ long and identical, which makes them highly susceptible to homologous recombination that can either expand or collapse the repeats [42]. Tandem repeats tend to be deleted rather than expanded unless deletion is selected against. This instability may be exacerbated by the deletion of the $x s e A$ gene in part of the population (Fig. 8a), which causes a hyper recombination phenotype in Escherichia coli. That the tandem triplication can be detected in Anabaena sp. WA102 over a span of two years, including in Anderson Lake, suggests that a selective pressure in the lake and in the culture may be maintaining the triplication. Key questions are whether the tandem repeat increases expression of the anaBCD operon and production of anatoxin-a, and whether elevated expression is under selection. Determining the selective pressure preserving the tandem repeat in the Anabaena sp. WA102 culture may illuminate the function of anatoxin-a in the environment.

\section{Closing the genome reveals details about genome architecture}

Long-read sequencing technology will increasingly allow for bacterial genomes to be assembled in a single step [43]. Closing the Anabaena sp. WA102 genome with as few as two PacBio SMRT cells demonstrates that it is pragmatic to use non-axenic environmental enrichments of targeted bacterial species in order to obtain their finished genomes. The long-read library (PacBio C6-P4 technology) used in this study yielded an average read length of $8.5 \mathrm{kbp}$, which is long enough to span long-repeat regions in most bacterial genomes including refractory genomes such as those of the bloom-forming cyanobacteria Anabaena and Microcystis [13, 44]. Greater access to long-read sequencing raises expectations for the quality of bacterial genome assembly and will yield new insight into the mobilome and structural variation in bacterial populations. The mobilome in many bacteria may be under-represented because mobile elements that are repeated throughout bacterial genomes cannot be assembled correctly with short-insert DNA libraries. Observing structural variation such as erosion of synteny (Fig. 6, Additional file 1: Figure S4) and accumulation of local repeats (Fig. 9) will enhance our understanding of bacterial evolution. In fact, short-insert libraries can be incorrectly assembled to suggest features that do not exist. An example of that is the missrepresentation of the $a n a B$ tandem repeat region in the Anabaena sp. WA102 genome (Fig. 9a). De novo assembly of short-insert genomic libraries is not sufficient to determine the number of replicons in a genome or overall gene order. Further, this method is liable to miss structural variants within a population, such as the fractional presence of an $x$ seA-bearing insertion (Fig. 8a). While short-read sequencing possesses distinct shortcomings in describing structural features of a genome, nearly all single-copy genes that make up the majority of a bacterial genome can be assembled from short-read Illumina sequencing runs (Table 1 and Fig. 2a).

\section{Predicted ecologic profile of Anabaena sp. WA102}

Mapping proteins from Anabaena sp. WA102 to the KEGG ortholog database indicates a metabolism acclimated to a nutrient-rich freshwater environment with 
ample sunlight. The inability to produce phycoerythrocyanin, produced by some related Anabaena, coupled with positive phototaxis and gas vesicle operons suggest that it competes for light by outmaneuvering other photosynthetic organisms and rising to the surface of the water to avoid niches with less green light. Competition experiments between other nitrogen-fixing autotrophs and Anabaena sp. WA102 could test these hypotheses. Freshwater cyanobacteria are known to secrete hydroxamatebased siderophores to chelate iron in water [45]. These siderophores, including those encoded by the $f h u$ genes in Anabaena sp. WA102, are then transported across the cell membrane by ferric-hydroxamate transporters [46]. Efficiently scavenging sulfur and iron would help maintain iron-sulfur clusters that are heavily used in nitrogen fixation and photosynthesis, so the predicted ability of Anabaena sp. WA102 to assimilate organic sulfur and oxidized iron from the lake environment may confer a growth advantage in some conditions over cyanobacteria lacking ssu, tau and fhu genes (Fig. 4).

\section{Evolution of the Anabaena sp. WA102 genome}

A genomic island and a complementary plasmid carrying novel genetic cargo (Additional file 1: Figure S7), tandem triplication of a promoter (Fig. 9), observed deletion of a $4 \mathrm{~kb}$ fragment of the genome (Fig. 8), the ubiquity of mobile elements (Fig. 2), and the nearly total absence of synteny with Anabaena sp. 90 (Fig. 6) suggest that the genome is in rapid flux. The potential for the genome to radically rearrange may allow Anabaena sp. WA102 to respond to gradual changes in the environment, such as climate change, if such changes offer the opportunity to adjust gene expression profiles. The increased availability of closed genomes as long-read sequencing becomes more widely used will allow us to quantify the rate of recombination in genomes in Anabaena and in other bacteria. It will then be possible to test hypotheses for the most prevalent mechanisms and drivers of genome remodeling.

More genomes from closely related species need to be finished with long-read sequencing. These genomes can then be arranged in an alignable tight genome cluster and assayed for gene family growth and loss, and for rearrangements [47]. Alternatively, resequencing metagenomes of the original environment of Anabaena sp. WA102 - Anderson Lake - at regular intervals is currently feasible. This approach would generate a regular time series record of differences in the population genome of Anabaena sp. WA102 in its native environment with nucleotide resolution.

\section{Methods}

\section{Sample collection}

$500 \mathrm{~mL}$ samples were collected from Anderson Lake, Washington State $(48.0190 \mathrm{~N}, 237.1963 \mathrm{~W})$ by the
Jefferson County Public Health Department during the 2012 and 2013 cyanobacterial toxic bloom seasons. Samples were collected at a depth of $0-0.5 \mathrm{~m}$ and may have included a dense windblown scum. Samples were shipped overnight on ice and several milliliters (depending on the sample density) were filtered through $0.2 \mu \mathrm{m}$ Pall Supor 200 and $1.2 \mu \mathrm{m}$-pore-size Whatman GF/C $24 \mathrm{~mm}$ diameter filters. Filters were stored at $-80{ }^{\circ} \mathrm{C}$ for later metagenomic sequencing. The culture was established upon sample arrival as described below.

\section{Culture establishment and maintenance}

A culture was established from a $0-0.5 \mathrm{~m}$ deep bloom sample collected from Anderson Lake on May 20th, 2013. The lake sample was concentrated tenfold by low-speed centrifugation (5,000 RCF). No buoyant cells were observed. Approximately $20 \mu \mathrm{L}$ of the concentrate was placed on a glass slide. Anabaena colonies were individually isolated by serially transferring the aliquot with an automatic pipette between at least five separate $50 \mu \mathrm{L}$ MilliQ water droplets on the glass slide. Colonies were considered to be isolated when no other cells or cell debris were visible in the surrounding water droplet under 200x magnification on a Zeiss brightfield microscope. Isolated colonies were placed in $200 \mu \mathrm{L}$ of BG-11 (i.e., BG-11 without nitrogen). BG-11 10 medium was prepared according to the Susan Golden Lab protocol (UC San Diego). One surviving colony was outgrown in BG- $11_{0}$ for several months, its identity was verified microscopically, and a single colony was again isolated into $200 \mu \mathrm{L}$ of BG-11. The outgrown colony was then maintained long-term in non-axenic batch culture in BG- $11_{0}$ under white fluorescent illumination of approximately $20 \mu \mathrm{Em}^{-2} \mathrm{~s}^{-1}$ at $24{ }^{\circ} \mathrm{C}$ with a light/dark cycle of $16 \mathrm{hr} / 8 \mathrm{hr}$. In addition to this culture, Dr. Mike Crayton from Pacific Lutheran University, Tacoma, Washington kindly shared a culture of Anabaena AL93 isolated in 1993 on BG-11 agar slants from American Lake, Pierce County, Washington State. It was maintained under the same conditions listed above but in BG-11 medium.

\section{LC-MS/MS}

Filters from lake samples were resuspended by dispersion in 500mL TNE buffer (50mM Tris- $\mathrm{HCl}$ (pH 7.5), $100 \mathrm{mM}$ $\mathrm{NaCl}, 0.1 \mathrm{mM}$ EDTA). Samples from resuspended filters or cultures were frozen and thawed for three cycles to release intracellular contents. Samples were centrifuged at 5,000 RCF for $5 \mathrm{~min}$, and the supernatant was removed for LC-MS/MS analysis. LC-MS/MS analysis was conducted using a hybrid quadrupole-time of flight instrument (AB Sciex TripleTOF, Foster City, CA) coupled to a Shimadzu Nexera LC-30a UHPLC system (Shimadzu, Columbia, MD). The DuoSpray ion source (AB Sciex, Foster City, CA) was operated in the positive electrospray 
ionization mode and the following settings were used: ion source gas 1, 40 psi; ion source gas 2, 50 psi; curtain gas, 25 psi; gas temperature, $550{ }^{\circ} \mathrm{C}$; and ion spray voltage, $5500 \mathrm{~V}$. The declustering potential (DP) was 80 $\mathrm{V}$ and the collision energy (CE) was set to $27 \mathrm{~V}$. The instrument was operated in positive ion polarity and highresolution product ion mode. Precursor ion selection was performed in the quadrupole operated at unit resolution. Precursor ions screened included: $\mathrm{m} / \mathrm{z} 166.1$ (anatoxina, $\mathrm{MH}^{+}, \mathrm{C}_{10} \mathrm{H}_{16} \mathrm{NO}^{+}$), m/z 168.1 (dihydro-anatoxin-a, $\mathrm{MH}^{+}, \mathrm{C}_{10} \mathrm{H}_{18} \mathrm{NO}^{+}$), m/z 180.1 (homoanatoxin-a, $\mathrm{MH}^{+}$, $\mathrm{C}_{11} \mathrm{H}_{18} \mathrm{NO}^{+}$) and $\mathrm{m} / \mathrm{z} 182.2$ (dihydro-homoanatoxin-a, $\mathrm{MH}^{+}, \mathrm{C}_{11} \mathrm{H}_{20} \mathrm{NO}^{+}$). Product ion mass spectral data were acquired using a scan range of $\mathrm{m} / \mathrm{z} 50-650$. Auto calibrations were performed prior to each LC-MS/MS run. Chromatographic separations were carried out using an Agilent Zorbax RRHD SB-18 column $(1.8 \mu \mathrm{m}$ particle size, $2.1 \times 150 \mathrm{~mm}$ ) held at $40{ }^{\circ} \mathrm{C}$. A binary solvent system was used consisting of water (solvent A, Fisher Optima LC/MS grade) and acetonitrile (solvent B, Fisher Optima LC/MS grade), both containing $0.1 \%$ formic acid (98\% pure, Sigma Aldrich). The following gradient was applied: 5 $\%$ B hold for 0.5 min then increase to $90 \%$ B within 5 min, reduce to $5 \%$ within $0.5 \mathrm{~min}$ and the hold for 5 min. Flow rate was $0.5 \mathrm{~mL} / \mathrm{min}$. Sample injection volume was $10 \mu \mathrm{L}$.

\section{DNA extraction and amplification}

DNA was extracted from cultures by concentrating the culture tenfold at 40,000 RCF and washing mucilage from the cell pellet with TNE buffer. The cell pellet was resuspended in TNE buffer and treated with a method from Neilan et al. [48] that had the following modifications. The protein fraction was removed with two 25:24:1 phenol/chloroform/isoamyl alcohol extractions followed by two chloroform extractions. Residual phenol was removed with a final diethyl-ether extraction. Total DNA from lake samples used for metagenome analysis was extracted from $1.2 \mu \mathrm{m}$-pore-size filters by macerating the filters with a pestle and extracting DNA as described.

\section{DNA sequencing}

Samples are listed (Table 1). Each Illumina library was prepared and sequenced at the Oregon State University Center for Gene Research and Biotechnology, Corvallis, Oregon. The Anabaena sp. WA102 culture was also sequenced using the PacBio C6-P4 long-read sequencing platform at the Washington State University Molecular Biology and Genomics Core, Pullman, Washington. Prior to PacBio sequencing, DNA fragments were size-selected on the BluePippin system (Sage Science) to enrich for reads longer than $8 \mathrm{kbp}$. Raw reads were collected from four PacBio SMRT cells.

\section{Draft genome binning}

Illumina metagenomes were assembled using idba version 1.1.1 assembler software [49] on a 64-bit Linux server with 500GB of RAM. Prior to assembly, any reads containing ambiguous basecalls ("N") were culled. The large chromosome from the Anabaena sp. 90 genome was used as a reference to guide assembly. Within idba, assemblies with kmer sizes ranging from 20nt to the sequence read length (100nt to 250nt) in 10nt increments were combined in the final assembly. Sequencing data from four PacBio SMRT cells for the Anabaena sp. WA102 culture was self-corrected, assembled, and polished using the Hierarchical Genome Assembly Process (HGAP) Pipeline at the Washington State University Molecular Biology and Genomics Core. Reads from original fastq files were mapped to the Illumina and PacBio assemblies using bwa version 0.7.5a-r405 [50]. Average coverage depth for each contig was calculated using samtools version 0.1.18 (r982:295) and the calc.coverage.in.bam.depth.pl script from the mmgenome package (https://github.com/ MadsAlbertsen/mmgenome) [51]. The mmgenome network.pl script generated a network of contigs based upon paired-end read data extracted from the bwa-generated SAM file. Bacterial and archaeal metagenome contigs were taxonomically classified using the PhylopythiaS+ support vector machine (SVM) classification software with only a contig fasta file and not a scaffold fasta file (https://github.com/algbioi/ppsp) [52]. 16S marker genes were detected in the contig file and used by PhylopythiaS+ to select an SVM training dataset automatically. Putative protein coding sequences were identified in each assembly fasta file using Prodigal version 2.6.2. To identify essential genes, putative protein sequences were aligned against a curated hmm database from the mmgenome package with the HMMER version 3.0 package (http:// hmmer.janelia.org/) [53]. A custom data generation shell script based on the data.generation.2.1.0.sh script from mmgenome was used to combine the above processes (https://github.com/russianconcussion/ data.analysis.scripts/blob/master/mmgenome.datagen.sh). Average coverage depth, network, taxonomic classification, and essential gene data for each assembly were imported into a data.frame structure in R. Finally, the mmgenome $\mathrm{R}$ package was used to generate a plot of genome clusters within the metagenomes, define and evaluate completeness of the clusters, and export well defined genome clusters as contigs in fasta format. Genome clusters in fasta format were annotated using Prokka version 1.11 [54].

\section{Finished Anabaena sp. WA102 genome analysis}

The finished Anabaena sp. WA102 genome was annotated using Prokka version 1.11 and the NCBI Prokaryotic Genome Annotation Pipeline after submission to 
Genbank. Non-ribosomal and polyketide synthesis gene clusters were annotated using the AntiSMASH webserver (http://antismash.secondarymetabolites.org/) [55]. The genome was scanned for prophages and genomic islands using the PHAST (http://phast.wishartlab.com/) and IslandViewer 3 (http://www.pathogenomics.sfu.ca/ islandviewer/) webservers [56, 57]. Insertion sequences were manually annotated with the IS Finder database [58]. BLASTN and CIRCOS were used to detect local alignments between Anabaena sp. WA102 and Anabaena sp. 90 and plot the corresponding similarities (http:// circos.ca/) [59, 60]. BLASTN, GenomicRanges, and CIRCOS were used to detect large repeat regions within the Anabaena sp. WA102 genome and map the Anabaena sp. WA102 Illumina assembly contigs to the finished genome [61]. Long and short repeat regions were also detected using RepeatScout to model repeat regions and RepeatMasker to annotate them (http://www.repeatmasker.org) [62]. Protein domains within the AnaG protein were identified with the SMART online protein domain database [63]. Whole genomes were aligned using Mauve 2.4.0 on default settings and Gepard 1.30.

\section{Comparative genomics among members of the Nostocaceae}

The putative protein-coding contents of Anabaena sp. WA102, Anabaena sp. AL93, Dolichospermum sp. AWQC131C, and Dolichospermum sp. AWQC310F was annotated using Prokka version 1.11. Protein content from Anabaena variabilis ATCC 29413, Anabaena sp. 90, Anabaena sp. PCC 7108, Anabaena cylindrica PCC 7122, Nostoc sp. PCC 7107, Nostoc sp. PCC 7120, and Nostoc sp. PCC 7524 were downloaded from Genbank. Proteincoding contents from each of the eleven genomes were used to build a phylogenomic tree. The bi-directional best BLASTP-hits method was used to identify orthologs in each genome [64]. These were clustered with the MCL algorithm and aligned with muscle [65, 66]. MCL inflation parameters were used as in the HAL paper ([67]; there were 13 inflation parameters), groups were selected starting from the highest moving to the lowest inflation parameter, selecting groups containg bidirectional best hits and no paralogs. BLAST settings were as found in [68], with an e-value threshold of 0.1. Protein alignments were masked with zorro to reduce noise from uninformative amino acid alignment positions and checked for a best fit among protein evolution models with ProtTest version $3.1[69,70]$. The best-fit protein evolution model was used in RAxML to generate the final tree, which was rooted within the Nostoc genus outgroup at Nostoc sp. 7107, in accordance with Shih et al. [10, 71]. Proteins were also mapped to the free KEGG database from 2011 and compared across metabolic pathways [72]. A grid that correlates highlighted KEGG comparisons with the phylogenomic tree described above was generated using the adephylo package in R [73].

\section{Accession numbers used in study}

Anabaena sp. WA102 [Genbank:CP011456-7], Anabaena sp. AL93 [Genbank:LJOU00000000], Dolichospermum sp. AWQC131C, Dolichospermum sp. AWQC310F, Anabaena variabilis ATCC 29413 [Genbank:NC007413], Anabaena sp. 90 [Genbank:NC_019427 and Genbank:CP 003285], Anabaena sp. PCC 7108 [Genbank:KB235895], Anabaena cylindrica PCC 7122 [Gen-bank:NC_019771], Nostoc sp. PCC 7107 [Genbank:NC_019676], Nostoc sp. PCC 7120 [Genbank:NC_003272], Nostoc sp. PCC 7524 [Genbank:NC_019684], Anabaena sp. 37 anatoxin-a region [Genbank:JF803645], Oscillatoria sp. PCC 6506 anatoxin-a region [Genbank:FJ477836], Cylindrospermum sp. PCC 7417 [Genbank:NC_019757], and WA25 metagenome sample [SRA:SRP066506].

\section{Additional file}

Additional file 1: Additional Figures 1-8 provide supporting information for the manuscript Brown et al., 2016, Structural and functional analysis of the finished genome of the recently isolated toxic Anabaena sp. WA102. (PDF $26100 \mathrm{~kb}$ )

\section{Abbreviations}

NRPS, Nonribosomal peptide synthase; PKS, Polyketide synthase; SMRT sequencing, Single-molecule real-time sequencing; ANI, Average nucleotide identity; LCB, Local colinear block

\section{Acknowledgements}

We thank Michael Dawson and Greg Thomason, Jefferson County Public Health, for collecting samples and providing field support. We thank Mike Crayton for donating the Anabaena sp. AL93 culture. We thank Lizbeth Seebacher at Washington State Department of Ecology for project support.

\section{Funding}

Funding for this study was provided by the Washington State Department of Ecology, Oregon State University Agricultural Experiment Station, and Mabel E. Pernot Trust. The acquisition of the qToF LC-MS/MS system used in this study was made possible 35by NIH grant S10RR027878.

\section{Availability of data and materials}

The datasets supporting the conclusions of this article are available in the NCB SRA repository (Original Illumina 100-bp reads from the Anabaena sp. WA102 culture: http://www.ncbi.nlm.nih.gov/sra/SRX1441873[accn]) and NCBI Genbank (The PacBio reference genome assembly of the Anabaena sp. WA102 culture: [http://www.ncbi.nIm.nih.gov/nuccore/CP011456.1] and [http://www. ncbi.nlm.nih.gov/nuccore/CP011457.1]). Both datasets can also be found linked under NCBI BioProject PRJNA282166 [http://www.ncbi.nlm.nih.gov/ bioproject/282166]. The Anderson Lake WA25 metagenome Illumina 100-bp read dataset will be publicly shared on the NCBI SRA database under the SRA run identifer SRR2936948, pending the publication of a separate research article containing a fuller analysis of the metagenome. The Nostocacaea phylogenomic tree in Fig. 3 and corresponding amino acid alignment matrix can be found on Treebase at [http://purl.org/phylo/treebase/phylows/study/ TB2:S19076].

\section{Authors' contributions}

NMB and TWD conceived and designed the experimental plan, with input from FJH and RSM, and wrote the manuscript with input from all authors. NMB conducted most of the experiments. RSM provided bioinformatic advice and analysis. JWS assisted with experiments. ZCL conducted the phylogenomic 
analysis; CSM and JTM conducted the mass spectrometry analysis. All authors have read and approved this manuscript.

\section{Competing interests}

The authors declare that they have no competing interests.

\section{Consent for publication}

Not applicable.

\section{Ethics approval and consent to participate}

Not applicable.

\section{Author details}

${ }^{1}$ Department of Microbiology, Oregon State University, 226 Nash Hall, 97331 Corvallis, OR, USA. ${ }^{2}$ Department of Chemistry, Oregon State University, 153 Gilbert Hall, 97331 Corvallis, OR, USA. ${ }^{3}$ Office of Environmental Public Health Sciences, Washington State Department of Health, 98504 Olympia, WA, USA ${ }^{4}$ Center for Genome Research and Biocomputing, Oregon State University, Corvallis, OR, USA.

\section{Received: 24 December 2015 Accepted: 12 May 2016}

Published online: 13 June 2016

\section{References}

1. Wacklin P, Hoffmann L, Komárek J. Nomenclatural validation of the genetically revised cyanobacterial genus Dolichospermum (Ralfs ex Bornet et Flahault) comb. nova. Fottea. 2009;9(1):59-64.

2. Golden JW, Yoon HS. Heterocyst development in Anabaena. Current Opin Microbiol. 2003;6(6):557-63.

3. Li X, Dreher TW, Li R. An overview of diversity, occurrence and toxin production of bloom-forming Dolichospermum (Anabaena) species. Harmful Algae. 2015. in press

4. Calteau A, Fewer DP, Latifi A, Coursin T, Laurent T, Jokela J, Kerfeld CA, Sivonen K, Piel J, Gugger M. Phylum-wide comparative genomics unravel the diversity of secondary metabolism in cyanobacteria. BMC Genomics. 2014;15(1):977.

5. Carmichael WW, Biggs DF, Gorham PR. Toxicology and pharmacological action of Anabaena flos-aquae toxin. Science. 1975;187(4176):542-4.

6. Carmichael WW, Biggs DF, Peterson MA. Pharmacology of anatoxin-a, produced by the freshwater cyanophyte Anabaena flos-aquae NRC-44-1. Toxicon. 1979;17(3):229-36.

7. Méjean A, Paci G, Gautier V, Ploux O. Biosynthesis of anatoxin-a and analogues (anatoxins) in cyanobacteria. Toxicon. 2014;91:15-22.

8. Rantala-Ylinen A, Känä $S$, Wang $H$, Rouhiainen $L$, Wahlsten M, Rizzi E, Berg K, Gugger M, Sivonen K. Anatoxin-a synthetase gene cluster of the cyanobacterium Anabaena sp. strain 37 and molecular methods to detect potential producers. Appl Environ Microbiol. 2011;77(20):7271-8.

9. Cadel-Six S, Iteman I, Peyraud-Thomas C, Mann S, Ploux O, Méjean A. Identification of a polyketide synthase coding sequence specific for anatoxin-a-producing Oscillatoria cyanobacteria. Appl Environ Microbiol. 2009;75(14):4909-12

10. Shih PM, Wu D, Latifi A, Axen SD, Fewer DP, Talla E, Calteau A, Cai F, de Marsac NT, Rippka R, et al. Improving the coverage of the cyanobacterial phylum using diversity-driven genome sequencing. Proc Nat Acad Sci. 2013;110(3):1053-8.

11. Kaneko T, Nakajima N, Okamoto S, Suzuki I, Tanabe Y, Tamaoki M, Nakamura Y, Kasai F, Watanabe A, Kawashima K, et al. Complete genomic structure of the bloom-forming toxic cyanobacterium Microcystis aeruginosa NIES-843. DNA Res. 2007;14(6):247-56.

12. Phillippy AM, Schatz MC, Pop M, et al. Genome assembly forensics: finding the elusive mis-assembly. Genome Biol. 2008;9(3):55.

13. Koren S, Harhay GP, Smith T, Bono JL, Harhay DM, Mcvey SD, Radune D, Bergman NH, Phillippy AM. Reducing assembly complexity of microbial genomes with single-molecule sequencing. Genome Biol. 2013;14(9):101.

14. Grigoriev A. Analyzing genomes with cumulative skew diagrams. Nucleic Acids Res. 1998;26(10):2286-90.

15. Wang H, Sivonen K, Rouhiainen L, Fewer DP, Lyra C, Rantala-Ylinen A, Vestola J, Jokela J, Rantasärkkä K, Li Z, et al. Genome-derived insights into the biology of the hepatotoxic bloom-forming cyanobacterium Anabaena sp. strain 90. BMC Genomics. 2012;13(1):613.

16. Griese M, Lange C, Soppa J. Ploidy in cyanobacteria. FEMS Microbiol Lett. $2011 ; 323(2): 124-31$
17. Eichhorn E, Van Der Ploeg JR, Leisinger T. Deletion analysis of the Escherichia coli taurine and alkanesulfonate transport systems. J Bacteriol. 2000;182(10):2687-95.

18. Köster W. ABC transporter-mediated uptake of iron, siderophores, heme and vitamin B 12. Res Microbiol. 2001;152(3):291-301.

19. Beckers G, Bendt AK, Krämer R, Burkovski A. Molecular identification of the urea uptake system and transcriptional analysis of urea transporter-and urease-encoding genes in Corynebacterium glutamicum. J Bacteriol. 2004;186(22):7645-52.

20. Mikulic M. Knock-out mutants of respiratory terminal oxidases in the cyanobacterium Anabaena sp. strain PCC 7120. PhD thesis, Universität Wien. 2013.

21. Yoshihara S, Ikeuchi M. Phototactic motility in the unicellular cyanobacterium Synechocystis sp. PCC 6803. Photochem Photobiol Sci. 2004;3(6):512-8.

22. Walsby A. Gas vesicles. Microbiol Rev. 1994;58(1):94.

23. Ting CS, Rocap G, King J, Chisholm SW. Cyanobacterial photosynthesis in the oceans: the origins and significance of divergent light-harvesting strategies. Trends Microbiol. 2002;10(3):134-42.

24. Swanson R, De Lorimier R, Glazer A. Genes encoding the phycobilisome rod substructure are clustered on the Anabaena chromosome: characterization of the phycoerythrocyanin operon. J Bacteriol. 1992;174(8):2640-7.

25. Pengelly JJL. Molecular characterisation of membrane transporters associated with saxitoxin biosynthesis in cyanobacteria: A dissertation submitted in partial fulfilment of the requirements for the award of doctor of philosophy (Ph. D). PhD thesis, UNSW, School of Biotechnology and Biomolecular Sciences. 2008.

26. Novichkov PS, Wolf YI, Dubchak I, Koonin EV. Trends in prokaryotic evolution revealed by comparison of closely related bacterial and archaeal genomes. J Bacteriol. 2009;191(1):65-73.

27. Eisen JA, Heidelberg JF, White O, Salzberg SL, et al. Evidence for symmetric chromosomal inversions around the replication origin in bacteria. Genome Biol. 2000;1(6):1-0011.

28. Spratt BG, Hanage WP, Feil EJ. The relative contributions of recombination and point mutation to the diversification of bacterial clones. Current Opin Microbiol. 2001;4(5):602-6.

29. Siguier P, Pérochon J, Lestrade L, Mahillon J, Chandler M. ISfinder: the reference centre for bacterial insertion sequences. Nucleic Acids Res. 2006;34(suppl 1):32-6.

30. De Palmenaer D, Siguier P, Mahillon J. IS4 family goes genomic. BMC Evol Biol. 2008;8(1):18

31. Reyes-Lamothe R, Nicolas E, Sherratt DJ. Chromosome replication and segregation in bacteria. Ann Rev Genet. 2012;46:121-43.

32. Wang H, Fewer DP, Holm L, Rouhiainen L, Sivonen K. Atlas of nonribosomal peptide and polyketide biosynthetic pathways reveals common occurrence of nonmodular enzymes. Proc Nat Acad Sci. 2014;111(25):9259-64.

33. Chase J, Richardson C. Escherichia coli mutants deficient in exonuclease VII. J Bacteriol. 1977;129(2):934-47.

34. Münch R, Hiller K, Grote A, Scheer M, Klein J, Schobert M, Jahn D. Virtual footprint and PRODORIC: an integrative framework for regulon prediction in prokaryotes. Bioinformatics. 2005;21(22):4187-9.

35. Lovett ST, Feschenko W. Stabilization of diverged tandem repeats by mismatch repair: evidence for deletion formation via a misaligned replication intermediate. Proc Nat Acad Sci. 1996;93(14) 7120-4.

36. Reams AB, Roth JR. Mechanisms of gene duplication and amplification. Cold Spring Harbor Perspect Biol. 2015;7:a016592.

37. Anderson RP, Roth JR. Tandem genetic duplications in phage and bacteria. Ann Rev Microbiol. 1977;31(1):473-505.

38. Reams AB, Neidle EL. Selection for gene clustering by tandem duplication. Annu Rev Microbiol. 2004;58:119-42.

39. Kondrashov FA, Rogozin IB, Wolf YI, Koonin EV. Selection in the evolution of gene duplications. Genome Biol. 2002;3(2):8-1.

40. Näsvall J, Sun L, Roth JR, Andersson DI. Real-time evolution of new genes by innovation, amplification, and divergence. Science. 2012;338(6105):384-7.

41. Schibler U, Sierra F. Alternative promoters in developmental gene expression. Ann Rev Genet. 1987;21(1):237-57.

42. Bi X, Liu LF. DNA rearrangement mediated by inverted repeats. Proc Nat Acad Sci. 1996;93(2):819-23. 
43. Koren S, Phillippy AM. One chromosome, one contig: complete microbial genomes from long-read sequencing and assembly. Current Opin Microbiol. 2015:23:110-20.

44. Yamaguchi H, Suzuki S, Tanabe Y, Osana Y, Shimura Y, Ishida K-I, Kawachi M. Complete genome sequence of Microcystis aeruginosa NIES-2549, a bloom-forming cyanobacterium from lake kasumigaura, japan. Genome Announcements. 2015;3(3):00551-15.

45. Wilhelm SW, Trick CG. Iron-limited growth of cyanobacteria: Multiple siderophore production is a common response. Limnol Oceanograph. 1994;39(8):1979-84

46. Stintzi A, Barnes C, Xu J, Raymond KN. Microbial iron transport via a siderophore shuttle: a membrane ion transport paradigm. Proc Nat Acad Sci. 2000;97(20):10691-6.

47. Puigbò $P$, Lobkovsky AE, Kristensen DM, Wolf $Y$, Koonin EV. Genomes in turmoil: quantification of genome dynamics in prokaryote supergenomes. BMC Biology. 2014;12(1):66.

48. Rochelle PA, et al. Environmental Molecular Microbiology: Protocols and Applications: Horizon Scientific Press; 2001, pp. 75-90.

49. Peng Y, Leung HC, Yiu SM, Chin FY. IDBA-UD: a de novo assembler for single-cell and metagenomic sequencing data with highly uneven depth. Bioinformatics. 2012;28(11):1420-8.

50. Li H, Durbin R. Fast and accurate short read alignment with Burrows-Wheeler transform. Bioinformatics. 2009;25(14):1754-60.

51. Albertsen M, Hugenholtz P, Skarshewski A, Nielsen KL, Tyson GW, Nielsen $\mathrm{PH}$. Genome sequences of rare, uncultured bacteria obtained by differential coverage binning of multiple metagenomes. Nat Biotechnol. 2013;31(6):533-8.

52. Gregor I, Dröge J, Schirmer M, Quince C, McHardy A. Phylopythias+: a self-training method for the rapid reconstruction of low-ranking taxonomic bins from metagenomes. 2014. arXiv preprint arXiv:1406.7123.

53. Eddy SR. Profile hidden Markov models. Bioinformatics. 1998;14(9):755-63.

54. Seemann T. Prokka: rapid prokaryotic genome annotation. Bioinformatics. 2014;30(14):2068-9.

55. Medema $M H$, Blin $K$, Cimermancic $P$, de Jager $V$, Zakrzewski $P$, Fischbach MA, Weber T, Takano E, Breitling R. antiSMASH: rapid identification, annotation and analysis of secondary metabolite biosynthesis gene clusters in bacterial and fungal genome sequences. Nucleic Acids Res. 2011;39(suppl 2):339-46.

56. Zhou Y, Liang Y, Lynch KH, Dennis JJ, Wishart DS. PHAST: a fast phage search tool. Nucleic Acids Res. 2011;39(Web Server issue):W347-52.

57. Dhillon BK, Chiu TA, Laird MR, Langille MG, Brinkman FS. IslandViewer update: improved genomic island discovery and visualization. Nucleic Acids Res. 2013:41(W1):129-32.

58. Siguier P, Filée J, Chandler M. Insertion sequences in prokaryotic genomes. Current Opin Microbiol. 2006;9(5):526-31.

59. Camacho C, Coulouris G, Avagyan V, Ma N, Papadopoulos J, Bealer K, Madden TL. BLAST+: architecture and applications. BMC Bioinformatics. 2009;10(1):421.

60. Krzywinski M, Schein J, Birol I, Connors J, Gascoyne R, Horsman D, Jones SJ, Marra MA. Circos: an information aesthetic for comparative genomics. Genome Res. 2009;19(9):1639-45.

61. Lawrence M, Huber W, Pages H, Aboyoun P, Carlson M, Gentleman R, Morgan MT, Carey VJ. Software for computing and annotating genomic ranges. PLoS Comput Biol. 2013;9(8):1003118.

62. Price $A L$, Jones NC, Pevzner PA. De novo identification of repeat families in large genomes. Bioinformatics. 2005;21(suppl 1):351-8.

63. Letunic I, Copley RR, Schmidt S, Ciccarelli FD, Doerks T, Schultz J, Ponting CP, Bork P. SMART 4.0: towards genomic data integration. Nucleic Acids Res. 2004;32(suppl 1):142-4.

64. Wolf $\mathrm{Yl}$, Koonin EV. A tight link between orthologs and bidirectional best hits in bacterial and archaeal genomes. Genome Biol Evol. 2012;4(12): 1286-94.

65. Enright AJ, Van Dongen S, Ouzounis CA. An efficient algorithm for large-scale detection of protein families. Nucleic Acids Res. 2002;30(7): 1575-84.

66. Edgar RC. MUSCLE: multiple sequence alignment with high accuracy and high throughput. Nucleic Acids Res. 2004;32(5):1792-7.

67. Robbertse B, Yoder RJ, Boyd A, Reeves J, Spatafora JW. Hal: an automated pipeline for phylogenetic analyses of genomic data. PLoS Currents. 2011;3:RRN1213.
68. Moreno-Hagelsieb G, Latimer K. Choosing blast options for better detection of orthologs as reciprocal best hits. Bioinformatics. 2008;24(3): 319-24.

69. Wu M, Chatteriji S, Eisen JA. Accounting for alignment uncertainty in phylogenomics. PLoS One. 2012;7(1):30288.

70. Darriba D, Taboada GL, Doallo R, Posada D. ProtTest 3: fast selection of best-fit models of protein evolution. Bioinformatics. 2011;27(8):1164-5.

71. Stamatakis A. RAxML-VI-HPC: maximum likelihood-based phylogenetic analyses with thousands of taxa and mixed models. Bioinformatics. 2006;22(21):2688-90.

72. Kanehisa M, Goto S. KEGG: kyoto encyclopedia of genes and genomes. Nucleic Acids Res. 2000;28(1):27-30.

73. Jombart T, Balloux F, Dray S. Adephylo: new tools for investigating the phylogenetic signal in biological traits. Bioinformatics. 2010;26(15): 1907-9.

\section{Submit your next manuscript to BioMed Central and we will help you at every step:}

- We accept pre-submission inquiries

- Our selector tool helps you to find the most relevant journal

- We provide round the clock customer support

- Convenient online submission

- Thorough peer review

- Inclusion in PubMed and all major indexing services

- Maximum visibility for your research

Submit your manuscript at www.biomedcentral.com/submit

C) Biomed Central 